\title{
The radial distribution of stars of different stellar generations in the globular cluster NGC 3201* (Research Note)
}

\author{
E. Carretta ${ }^{1}$, A. Bragaglia ${ }^{1}$, V. D’Orazi ${ }^{2}$, S. Lucatello ${ }^{2,3,4}$, and R. G. Gratton ${ }^{2}$ \\ 1 INAF-Osservatorio Astronomico di Bologna, via Ranzani 1, 40127 Bologna, Italy \\ e-mail: eugenio. carretta@oabo.inaf.it \\ 2 INAF-Osservatorio Astronomico di Padova, Vicolo dell'Osservatorio 5, 35122 Padova, Italy \\ 3 Excellence Cluster Universe, Technische Universität München, Boltzmannstr. 2, 85748 Garching, Germany \\ 4 Max-Planck-Institut für Astrophysik, 85741 Garching, Germany \\ Received 17 May 2010 / Accepted 24 June 2010
}

\section{ABSTRACT}

\begin{abstract}
We study the radial distribution of stars from different stellar generations in the globular cluster NGC 3201. From recently published multicolour photometry, a radial dependence of the location of stars on the giant branch was found. We coupled the photometric information to our sample of 100 red giants with $\mathrm{Na}, \mathrm{O}$ abundances and with known classification as first or second-generation stars. We find that giants stars of the second generation in NGC 3201 show a tendency to be more centrally concentrated than stars of the first generation, supporting less robust results from our spectroscopic analysis.
\end{abstract}

Key words. stars: abundances - stars: atmospheres - stars: population II - globular clusters: general globular clusters: individual: NGC 3201

\section{Introduction}

Chemical inhomogeneities in light elements $(\mathrm{C}, \mathrm{N}, \mathrm{O}, \mathrm{F}, \mathrm{Na}$, $\mathrm{Al}, \mathrm{Mg}$, and even $\mathrm{Si}$ ) are intrinsic to globular clusters (GCs; see Gratton et al. 2004, for an extensive review and references). In particular, the striking anticorrelation between $\mathrm{Na}$ and $\mathrm{O}$ abundances in GC red giant branch (RGB) stars discovered and studied by the Texas-Lick group (see Kraft 1994, for a review on those pioneering efforts) is the most notable signature observed with high-resolution spectroscopy. The pivotal discovery of the $\mathrm{Na}-\mathrm{O}$ (and the $\mathrm{Mg}-\mathrm{Al}$ ) anticorrelation in unevolved cluster stars (Gratton et al. 2001) led to the unambiguous conclusion that stars of different generations co-exist in the currently observed GCs. The reason is that the high temperatures required for proton-capture reactions (Denisenkov \& Denisenkova 1989; Langer et al. 1993) to produce matter depleted in $\mathrm{O}, \mathrm{Mg}$ and enriched in $\mathrm{Na}, \mathrm{Al}$ are never reached in the interior of low-mass stars. (Temperature in excess of 25 or $70 \times 10^{6} \mathrm{~K}$ are required for the $\mathrm{NeNa}$ and $\mathrm{MgAl}$ cycles, respectively.) As a consequence, the observations by Gratton et al. (later confirmed by e.g., Cohen et al. 2002; Carretta et al. 2004; D'Orazi et al. 2010) require the existence of a previous stellar generation of more massive stars with higher internal temperatures sufficient to activate the necessary nucleosynthesis, providing the ejecta from which the second-generation stars formed. Dilution processes with pristine gas left in the cluster may then reproduce the whole length of the Na-O anticorrelation (see Pranztos \& Charbonnel 2006; see however Gratton \& Carretta 2010).

The Na-O anticorrelation is so widespread among GCs (see e.g., Carretta et al. 2009a,b) that this feature is probably associated to the very same mechanism of cluster formation

$\star$ Based on observations collected at ESO telescopes under programme 073.D-0211.
(Carretta 2006) and may be considered the main criterion for distinguishing bona fide GCs (Carretta et al. 2010) and other type of clusters, regardless of their old ages or even high mass (e.g. NGC 6791, Bragaglia 2010, in prep.).

However, while the overall pattern of the chemical composition of first and second-generation GC stars is currently well assessed, several issues are still left open, the main one being the nature of the actual polluters, either intermediate-mass asymptotic giant branch stars (IM-AGBs, Ventura et al. 2001) or fastrotating massive stars (FRMSs, Decressin et al. 2007).

One of the main questions concerns the possible link between chemical signature and dynamical evolution of different stellar generations in GCs. This issue is puzzling and still poorly explored in a systematic way by theoretical models. Models where a cooling-flow feeds gas enriched in IM-AGB ejecta to form second-generation stars (D'Ercole et al. 2008), intrinsically predict that this generation should be more centrally concentrated, since the gas is re-collected at the cluster centre. On the other hand, also second-generation stars formed by matter polluted by FRMS are expected to be more centrally concentrated. They should have the same radial distribution as their progenitors, which are assumed to be born near the cluster centre, since they are massive objects.

Even if the second generation formed at the cluster centre, there is the action of the dynamical evolution over a Hubble time to be considered. For instance, Decressin et al. (2008) compute that the second-generation stars are progressively spread out by dynamical encounters. As a result, the radial distributions of first and second-generation stars can no longer be distinguished from their dynamics alone at the present time. In this Note we combine information from our ongoing FLAMES survey of chemical abundances in giants in GCs (see Carretta 2006) with newly derived photometry for the GC NGC 3201 




Fig. 1. Cumulative distributions of the primordial $\mathrm{P}$ (blue dotted line), the intermediate I (red solid line), and the extreme E stellar components (green dashed line) in NGC 3201 as a function of the distance from the cluster centre. The results of the comparison between the distributions using a K-S test are also listed.

(Kravtsov et al. 2009, 2010), and we provide new insight into the radial distribution of first and second-generation stars in this $\mathrm{GC}$, whose relaxation time at half-mass radius is about $1.6 \mathrm{Gyr}$ (Harris 1996).

\section{Radial distribution of $P, I$, and $E$ stars in NGC 3201}

In Carretta et al. (2009a) we showed that it is possible to separate a stellar component of first-generation (or primordial, P) stars in all the GCs observed. The remaining second-generation stars can be further separated into intermediate (I) and extreme (E) components, according to the degree of O-depletion and $\mathrm{Na}$ enhancement along the $\mathrm{Na}-\mathrm{O}$ anticorrelation.

We also tried to study the radial distribution of P, I, and E stars from the total sample of almost 2000 RGB stars in $19 \mathrm{GCs}$, but concluded that the merged sample is not the best option. Owing to the physical limitations of the FPOSS positioner of the FLAMES fibres and to different mass, concentration, and size of the GCs, we were not able to sample cluster regions that are dynamically equivalent in all objects. As a consequence, the cumulative distributions from the whole sample in Carretta et al. (2009a) may be biased.

This bias is partly reduced when looking at an individual cluster. As an example, we select NGC 3201, since it hosts a good fraction of each component among the 100 stars with $\mathrm{Na}$, $\mathrm{O}$ abundances $(P=35 \pm 6 \%, I=56 \pm 7 \%$, and $E=9 \pm 3 \%$, Carretta et al. 2009a). This cluster is also the subject of a recent publication advocating inhomogeneity of its stellar population on a photometric basis (Kravtsov et al. 2010, see below).

In Fig. 1 we show the cumulative distributions of radial distances for stars of the P, I, and E components in NGC 3201. Although it is possible to see a trend toward second-generation stars being more centrally concentrated (and pregressively more the E than the I component), the distributions show no statistically significant differences if we apply a Kolmogorov-Smirnov (K-S) test.

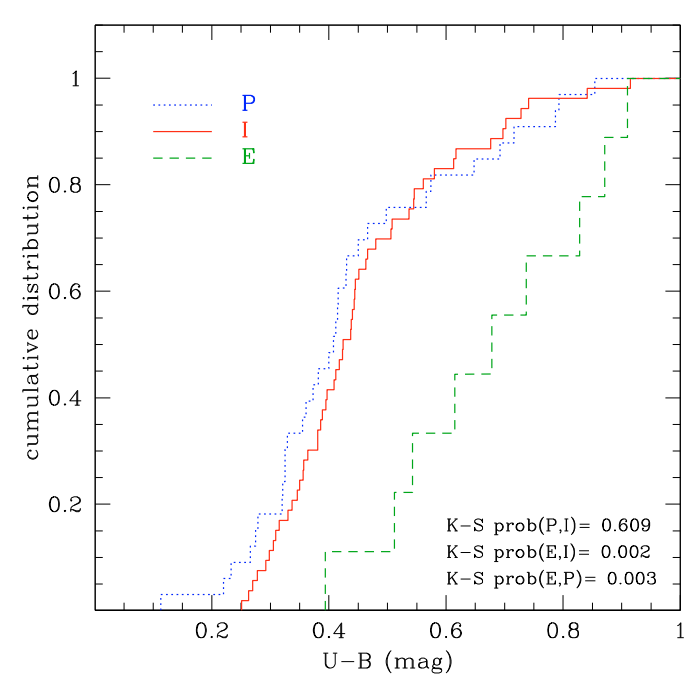

Fig. 2. Cumulative distributions of $U-B$ colours of $\mathrm{P}$ (blue dotted line), I (red solid line), and E (green dashed line) stars in NGC 3201. The results of the comparison between the distributions using a K-S test are also listed.

Much more significant are the differences in colour observed along the RGB for the $\mathrm{P}, \mathrm{I}$, and E populations, in particular when using the $U-B$ colour. We do not have $U$ in our photometric database, so we cross-identified our stars with the multi-colour photometry of more than 12000 stars in NGC 3201 by Kravtsov et al. (2009). We used software written by P. Montegriffo at the Bologna Observatory and found 138 stars (out of 150) in common between the two samples. Among these, 95 objects $^{1}$ can be classified as P, I, or E RGB stars, and their distributions in $U-B$ colour are shown in Fig. 2.

In this case, while the $\mathrm{P}$ and I distributions are not statistically different, there is no doubt that the E stars have redder colours than the other two components, at the very high level of confidence. This is not a new result: Marino et al. (2008), Yong et al. (2008), and Carretta et al. (2009a) convincingly showed that in M 4 and NGC 6752, Na-poor/O-rich/N-poor first-generation stars are segregated on the RGB into a narrow strip to the blue ridge of the branch, either in the $U, U-B$ colour-magnitude diagram (CMD) or in the $u, u-b$ CMD from Strömgren photometry. Second-generation stars (I+E, Na-rich/O-poor, and N-rich) are instead spread out to the red of the RGB. This different location is the effect of $\mathrm{N}$ abundances, affecting $U-B$ and $u-b$ colour indexes through the formation of $\mathrm{NH}$ and $\mathrm{CN}$ molecular bands falling into the windows of the ultraviolet filters response.

Figure 2 simply confirms this behavious for yet another GC, by coupling colours and $\mathrm{Na} / \mathrm{O}$ abundances. However, the novelty presented in this Note is that we can exploit this property to get independent insight into the radial distributions of stars in NGC 3201, which is completely free of any bias in the spectroscopic sample.

Kravtsov et al. (2010) find signs of multiple sequences among subgiant branch stars in this GC, with stars at different magnitude levels primarily lying at different radial distances,

1 We required both $\mathrm{Na}$ and $\mathrm{O}$ values for the classification, so stars for which one of the two is not available were not considered. However, a less strict classification in first and second-generation (without distinction between $\mathrm{I}$ and $\mathrm{E}$ ) is possible whenever $\mathrm{Na}$ is measured (see e.g. Bragaglia et al. 2010). 


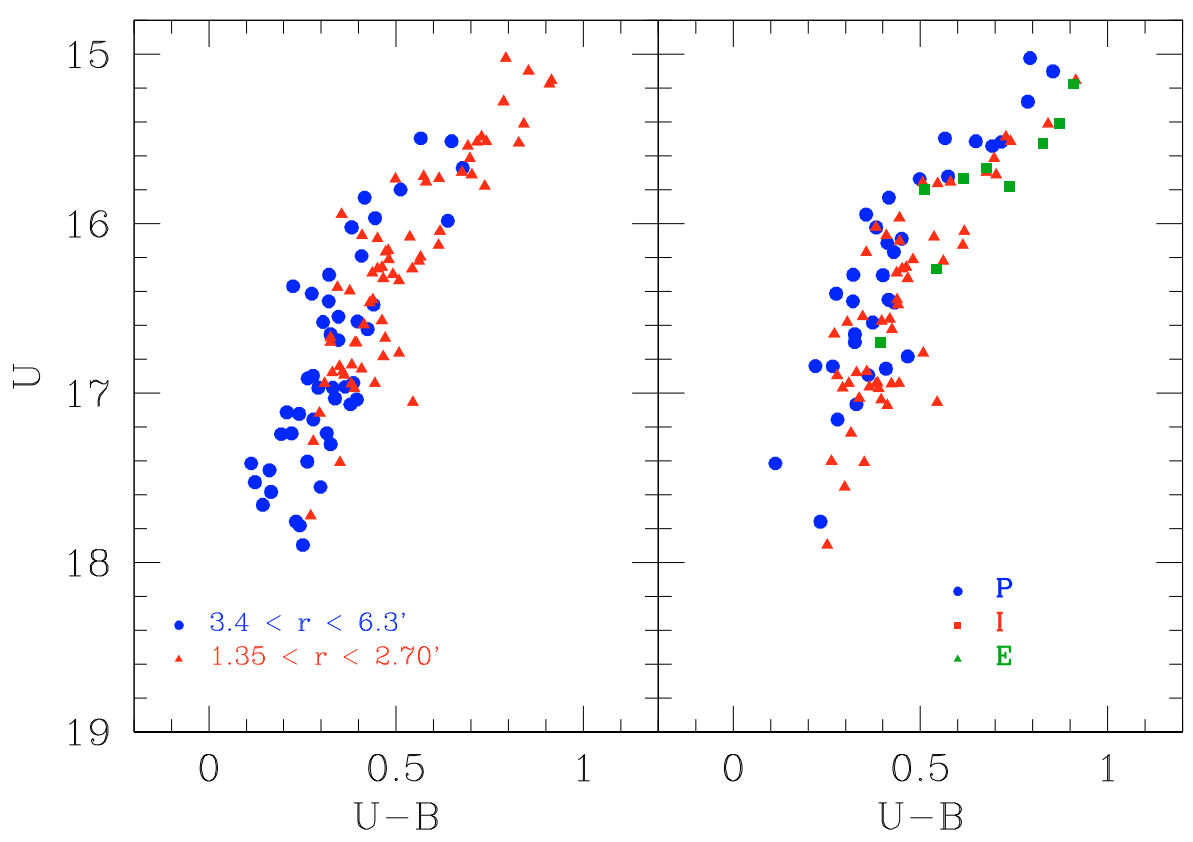

Fig. 3. Left panel: $U, U-B$ CMD (Kravtsov et al. 2009) for RGB stars of NGC 3201 in common with Carretta et al. (2009a,b). Blue circles and red filled triangles corresponds to the two different intervals in radial distances used in Kravtsov et al. (2010). Right panel: the same CMD using stars in common, but with symbols are colour-coded according to the $\mathrm{P}$ (blue circles), I (red triangles), and $\mathrm{E}$ (green squares) components of first and secondgeneration stars. with a high level of statistical significance. Additionally, a similar dependence on the radial distances is observed among RGB stars, with the bluer stars in $U-B$ sistematically located towards outer cluster regions (see Kravtsov et al. 2010, their Fig. 3).

We show in Fig. 3 (left panel) the $U, U-B$ CMD for all the 138 stars of our sample in NGC 3201 in common with Kratsov et al. The colour coding identifies the same two intervals in radial distances used by them and reflects the trend noted in the radial distribution. In the right panel, however, the stars in common $^{2}$ are simply colour-coded according to their status of P, I, or E. The comparison between the two plots immediately tells us that second-generations stars in NGC 3201 must also be those more concentrated toward the cluster centre, according to their location on the CMD.

This result supports what we find from our sample only (see Fig. 1), but is now more robust, because it is anchored to a much larger sample (almost 500 stars with $U B V I$ photometry with respect to about 100 RGB stars with $\mathrm{Na}, \mathrm{O}$ abundances).

In conclusion, we find that second-generation stars are most likely more concentrated toward the inner cluster regions in NGC 3201. Apparently, it seems that even if the relaxation time of NGC 3201 is much shorter than its lifetime, some trace of the initial formation process might still be recognizable. Interestingly, Norris \& Freeman (1979) found evidence of the same effect in another cluster, 47 Tucane: they determined that the $\mathrm{CN}$-strong stars, i.e., the ones that we now attribute to the second generation, are more centrally located.

While our finding does not help differentiate between scenarios where the pollution comes either from AGB or FRMS, it offers another piece of evidence that is useful for reconstructing the complex puzzle of multiple stellar populations in GCs.
Acknowledgements. This work was partially funded by the Italian MIUR under PRIN 2003, 2007, and by the grant INAF 2005 "Experimenting nucleosynthesis in clean environments". This research made use of the SIMBAD database (in particular Vizier), operated at the CDS, Strasbourg, France, and of NASA's Astrophysical Data System.

\section{References}

Bragaglia, A., Carretta, E., Gratton, R., et al. 2010, A\&A, 519, A60 Carretta, E. 2006, AJ, 131, 1766

Carretta, E., Gratton, R. G., Bragaglia, A., Bonifacio, P., \& Pasquini, L. 2004, A\&A, 416, 925

Carretta, E., Bragaglia, A., Gratton, R. G., et al. 2006, A\&A, 450, 523

Carretta, E., Bragaglia, A., Gratton, R. G., et al. 2009a, A\&A, 505, 117

Carretta, E., Bragaglia, A., Gratton, R. G., \& Lucatello, S. 2009b, A\&A, 505, 139 (Paper VIII)

Carretta, E., Bragaglia, A., Gratton, R. G., et al. 2010, A\&A, 516, 55

Cohen, J. G., Briley, M. M., \& Stetson, P. B. 2002, AJ, 123, 2525

Decressin, T., Meynet, G., Charbonnel, C. Prantzos, N., \& Ekstrom, S. 2007, A\&A, 464, 1029

Decressin, T., Baumgardt, H., \& Kroupa, P. 2008, A\&A, 492, 101

Denisenkov, P. A., \& Denisenkova, S. N. 1989, A. Tsir., 1538, 11

D'Ercole, A., Vesperini, E., D’Antona, F., McMillan, S. L. W., \& Recchi, S. 2008, MNRAS, 391, 825

D'Orazi, V., Lucatello, S., Gratton, R., et al. 2010, ApJ, 713, L1

Gratton, R. G., \& Carretta, E. 2010, A\&A, accepted

Gratton, R. G., Bonifacio, P., Bragaglia, A., et al. 2001, A\&A, 369, 87

Gratton, R. G., Sneden, C., \& Carretta, E. 2004, ARA\&A, 42, 385

Harris, W. E. 1996, AJ, 112, 1487

Kraft, R. P. 1994, PASP, 106, 553

Kravtsov, V., Alcaino, G., Marconi, G., \& Alvarado, F. 2009, A\&A, 497, 371

Kravtsov, V., Alcaino, G., Marconi, G., \& Alvarado, F. 2010, A\&A, 512, L6

Langer, G. E., Hoffman, R., \& Sneden, C. 1993, PASP, 105, 301

Marino, A., Villanova, S., Piotto, G., et al. 2008, A\&A, 490, 625

Norris, J., \& Freeman, K. C. 1979, ApJ, 230, L179

Prantzos, N., \& Charbonnel, C. 2006, A\&A, 458, 135

Ventura, P. D’Antona, F., Mazzitelli, I., \& Gratton, R. 2001, ApJ, 550, L65

Yong, D., Grundahl, F., Johnson, J. A., \& Asplund, M. 2008, ApJ, 684, 1159

\footnotetext{
2 At the faintest magnitudes some stars are missing in the right panel, in particular on the blue side. This happens because of the increased difficulty in measuring reliable equivalent widths for faint and warm stars. In Carretta et al. (2009a), only stars with both $\mathrm{Na}$ and $\mathrm{O}$ abundances are attributed to one of the P, I, E populations.
} 